\title{
A comparative study of American ginseng (spp. Panax quinquefolius) and Donepezil on working memory in rats using scopolamine induced disruption of memory
}

\author{
Shrikrishna Shende*, Kalbhairav M. Shinde, Abhijit V. Tilak, Bhalachandra T. Rane, \\ Siddhi Raveendran, Sanjay A. Dabhade
}

Department of Pharmacology, Dr. D. Y. Patil Medical College, Pimpri, Pune 411018, Maharashtra, India

Received: 30 January 2017 Accepted: 06 February 2017

*Correspondence to:

Dr. Shrikrishna Shende, Email: drkrish999@gmail.com

Copyright: (C) the author(s), publisher and licensee Medip Academy. This is an openaccess article distributed under the terms of the Creative Commons Attribution NonCommercial License, which permits unrestricted noncommercial use, distribution, and reproduction in any medium, provided the original work is properly cited

\begin{abstract}
Background: Treatment of Alzheimer's disease (AD) includes acetylcholinesterase inhibitors like Donepezil. American ginseng has been postulated to enhance learning and memory owing to its antiapoptotic activity as well as inhibition of formation of $\beta$ amyloid plaque. Scarce data is available on effect of combination of these two drugs. Hence, we have conducted this study to evaluate the same. Aim and objectives of the study were to evaluate the role of American ginseng on working memory in rats and to compare the effect of American ginseng with Donepezil on working memory in rats.

Methods: Sprague-Dawley rats of either sex, weighing 150-200g were used and obtained from animal house of Dr. D. Y. Patil Medical College, Hospital and Research Centre, Pimpri, Pune. To study the effects of the drugs on working memory in rats, they were trained for conditioned avoidance response (CAR) using Cook's pole climbing apparatus. The method of Fellow and Cook was used.

Results: In the group C (i.e. the control group), $50 \%$ of rats showed the retention of CAR. In the group S (i.e. only scopolamine group), only $20 \%$ of rats showed the retention of CAR. In the group $\mathrm{S}+\mathrm{AG}$ (i.e. scopolamine and American ginseng), $60 \%$ of rats showed the retention of CAR. In the group S+ D (i.e. scopolamine and Donepezil) $70 \%$ of rats showed retention of CAR. In the group $\mathrm{S}+\mathrm{D}+\mathrm{AG}$ (i.e. the combination group of scopolamine, Donepezil and American ginseng), $80 \%$ of rats showed the retention of CAR.

Conclusions: From this study we can conclude that American ginseng and Donepezil have comparable effects in retention of working memory. The combination of American ginseng and Donepezil can have additive effect on the retention of working memory.
\end{abstract}

Keywords: American ginseng, Conditioned avoidance response (CAR), Donepezil, Scopolamine

\section{INTRODUCTION}

Dementia, a syndrome of many causes, affects many people worldwide and can be defined as an acquired deterioration in the cognitive abilities that impairs the successful performance of activities of daily living. Memory is the most common cognitive ability lost with dementia. Amnesia occurs when the portion of the brain responsible for retrieving the stored memories is somehow compromised. ${ }^{1}$

There are a number of clinical conditions which can affect the cognition. These are depression, schizophrenia, multiple sclerosis, vasculitis and the commonest being $\mathrm{AD}$, Parkinsonism and alcoholism, with $\mathrm{AD}$ being the most common. $\mathrm{AD}$ is the most common cause of dementia in the elderly, with a prevalence that doubles every five years after the age of 60. According to National Institute of Health, an estimated 8.5 million Americans will develop AD by the year $2030 .^{2}$

$\mathrm{AD}$ is a progressive neurodegenerative disease whose pathogenesis involves both cholinergic and noncholinergic mechanisms. The loss of cholinergic neurons occurs primarily in the cortex and the 
hippocampus, the brain structures that play important roles in the memory and cognitive function.

Learning has been associated with the increased activity of cholinergic neurons and significant cortical acetylcholine (Ach) augmentation, which appears to act on postsynaptic muscarinic receptors. ${ }^{5}$ Furthermore, increases in the cortical ACh levels are observed following learning, which also supports the notion that ACh release contributes to the memory consolidation. ${ }^{3}$

Various strategies have been investigated to attempt to increase the cholinergic function in $\mathrm{AD}$, but the use of cholinesterase (ChE) inhibitors has been the most clinically successful. Donepezil has been in the market the longest, and more published clinical trials exist for Donepezil than for the newer drugs. Donepezil therapy has been shown to improve the behavioural symptoms in the patients with mild to moderate AD. ${ }^{2}$

The standardized American ginseng (Panax quinquefolius) extract preparation has been found to exhibit multifaceted therapeutic effects that include effects on neurodegenerative diseases, cancer, cardiovascular diseases, anti-inflammatory effects, antiallergic effects, erectile dysfunction and psychiatric disorders. ${ }^{4}$ Human studies have shown that American ginseng root extract (Ginsenosides Cereboost ${ }^{\mathrm{TM}}$ ) 200 $\mathrm{mg} /$ day significantly has shown improvements in the cognitive performance and increased calmness in healthy young adults. Amongst all doses compared (100,200,400 $\mathrm{mg}$ ), the most robust effects were observed for $200 \mathrm{mg}$ dose. $^{5}$

In view of the various reports indicating that supplementation with various herbal and allopathic drugs may enhance the learning and memory, and the fact that there are hardly any published trial reports on the comparative evaluation of various formulations used in India, the present study was undertaken to evaluate and to compare the role of American ginseng with Donepezil by using established model producing amnesia: Scopolamine induced disruption of conditioned avoidance response. ${ }^{6-8}$

\section{METHODS}

\section{Animals used}

The animals used in the study were experimentally naive albino rats. They were selected as per the following criteria:

- $\quad$ Species: Rattus norvegicus

- Strain: Sprague dawley

- Sex: Male/Female

- $\quad$ Body Weight range: $150-200 \mathrm{~g}$

The study was approved by the Institutional Animal Ethics Committee (IAEC).

\section{Study drugs}
I. American ginseng
II. Donepezil

\section{Chemicals/drugs/vehicles used}

I. Donepezil suspension

II. American ginseng suspension

III. Scopolamine hydrobromide suspension

IV. Double distilled water as vehicle

\section{Plan of the study}

To study the effects of study drugs on the working memory in rats, the animals were tested for retention of CAR (Conditioned Avoidance Response).

\section{Conditioned avoidance response (CAR)}

\section{Screening}

The rats were trained for CAR by using Cook's pole climbing apparatus. The method of Fellow and Cook was used with some modification. Each rat was allowed to acclimatize for two minutes, and was then exposed to a buzzer noise. After 5 seconds of putting on the buzzer, mild electric shocks were given through the stainless steel grid floor. The magnitude of the voltage was adequate (5$10 \mathrm{~V})$ to stimulate the rat to escape from the floor and climb the pole. As soon as the rat climbed the pole, both the buzzer and the foot shocking were switched off. At least 10 such trials were given to each rat at an interval of 1 minute per day, for 10 days. After about ten days training schedule, most of the rats learned to climb the poles within five seconds of starting the buzzer, thus avoiding the electric foot shocks. Rats avoiding the foot shocks in all ten out of ten trials were considered to have developed CAR for further experiments. ${ }^{9,10}$

\section{Pretreatments}

\section{Scopolamine induced disruption in memory}

Rats trained for CAR received scopolamine hydrobromide in a dose of $0.5 \mathrm{mg} / \mathrm{kg}$ by intraperitoneal route before administration of study drugs. This is known to produce amnesia which will be used to evaluate the effect of study drugs on learning and memory. ${ }^{11}$

\section{Study drug administration}

I. Donepezil: Donepezil was given in a dose of 0.32 $\mathrm{mg} / \mathrm{kg}$ by intraperitoneal route for eight days in the animals after training for CAR.

II. American ginseng: American ginseng was given in a dose of $18 \mathrm{mg} / \mathrm{kg}$ by oral route for eight days in the animals after training for CAR. ${ }^{12}$

III. Scopolamine: Scopolamine was given in a dose of $0.5 \mathrm{mg} / \mathrm{kg}$ by intraperitoneal route, 20 minutes 
before the administration of study drug in the animals after training for CAR. ${ }^{12}$

IV. Double distilled water was used as a vehicle for dissolving both study drugs and was administered as a vehicle in the control group orally.

\section{Grouping}

The animals were divided into five different groups of 10 rats each, after training for CAR. The animals received drugs either by intraperitoneal route or oral route, depending on the group.

The rats were divided into following groups:

In each group at the end of eighth day (i.e. on the ninth day) rats were evaluated for changes in the retention of CAR.

1. Control $(C)$ : Distilled water given orally for eight days, which was used as the vehicle for study drugs, and served as control.

2. Scopolamine (S): Received scopolamine $(0.5 \mathrm{mg} / \mathrm{kg}$ intraperitoneally) 20 minutes before each test runs on the apparatus.

3. S+American Ginseng $(A G)$ : Received scopolamine $(0.5 \mathrm{mg} / \mathrm{kg}$ intraperitoneally) 20 minutes before each test run on the apparatus, and American ginseng (18mg/kg orally) for 8 days.

4. S+Donepezil (D): $\quad$ Received scopolamine $(0.5 \mathrm{mg} / \mathrm{kg}$ intraperitoneally) 20 minutes before each test run on the apparatus and Donepezil $(0.32$ $\mathrm{mg} / \mathrm{kg}$ by intraperitoneally route) for 8 days.

5. $S+D+A G$ : Received scopolamine $(0.5 \mathrm{mg} / \mathrm{kg}$ intraperitoneally) $20 \mathrm{~min}$ before each test run on the apparatus, American ginseng and Donepezil in above mentioned doses for 8 days.

All the cages were tagged as per the groups mentioned above. All the rats were confined to their respective cages.

On day nine, all rats were tested to see if they had retained the CAR. After two minutes of acclimatization period, each rat was exposed to the buzzer for five seconds. Ten such trials were given at an interval of one minute, without giving any foot shock. Rats responding by climbing the pole when exposed to the buzzer noise were considered to have retained the CAR.

\section{Methods of disposal post-experiment}

No animals were sacrificed. They were kept separately from the colony for around four weeks. After that, the animals were checked for normal parameters.

\section{Data analysis}

The data was compiled and analysed using Primer of Biostatistics Version 7.0.
- Appropriate statistical tests as one way ANOVA were applied wherever required. The multiple comparison done by Bonferroni adjusted t-test between data was considered statistically significant only if $\mathrm{P}<0.05$.

Assessment was done by comparing the results obtained in the scopolamine pre-treated, scopolamine + American ginseng pre-treated, scopolamine + Donepezil pretreated, scopolamine + American ginseng + Donepezil pre-treated, and with those in the control group. Assessment was also done by comparing scopolamine + American ginseng pre-treated, scopolamine + donepezil pre-treated, scopolamine + Donepezil + American ginseng pre-treated, with those of the scopolamine group.

Comparison between scopolamine + American ginseng and scopolamine + Donepezil group, was also done. We also compared the combination groups with the groups containing drugs alone i.e. comparison of scopolamine + American ginseng and scopolamine + Donepezil group was done with scopolamine + Donepezil + American ginseng group.

\section{RESULTS}

As per the plan, the study was carried out in five different groups.

Table 1: Percentage of rats showing retention of CAR in scopolamine group.

\begin{tabular}{|lll|}
\hline Groups & Yes (Retention of CAR \%) & Mean \pm SD \\
\hline C & $50 \%$ & $5 \pm 1.555$ \\
\hline S & $20 \%$ & $2 \pm 0.9428$ \\
\hline S+AG & $60 \%$ & $6 \pm 1.9444$ \\
\hline S+D & $70 \%$ & $7 \pm 1.054$ \\
\hline S+D+AG & $80 \%$ & $8 \pm 1.491$ \\
\hline
\end{tabular}

In the group $\mathrm{C}$ (i.e. the control group), $50 \%$ of rats showed the retention of CAR.

In the group S (i.e. only scopolamine group), only $20 \%$ of rats showed the retention of CAR.

In the group $\mathrm{S}+\mathrm{AG}$ (i.e. scopolamine and American ginseng), $60 \%$ of rats showed the retention of CAR.

In the group S+ D (i.e. scopolamine and Donepezil) $70 \%$ of rats showed retention of CAR.

In the group $\mathrm{S}+\mathrm{D}+\mathrm{AG}$ (i.e. the combination group of scopolamine, Donepezil and American ginseng), $80 \%$ of rats showed the retention of CAR.

- When group $\mathrm{S}$ is compared with group $\mathrm{C}$, decrease in the retention of CAR was statistically significant as compared to the group $\mathrm{C}$.

- When group $\mathrm{S}+\mathrm{AG}$ is compared with group $\mathrm{S}$, increase in the retention of CAR in the $S+G$ group 
was statistically significant as compared to the group S.

- When group $\mathrm{S}+\mathrm{AG}$ is compared with group $\mathrm{C}$, increase in the retention of CAR was not statistically significant as compared to the group $\mathrm{C}$.

- When group $\mathrm{S}+\mathrm{D}$ is compared with group $\mathrm{S}$, increase in the retention of CAR was statistically significant as compared to the group $\mathrm{S}$.

- When group $\mathrm{S}+\mathrm{D}$ is compared with group C, increase in the retention of CAR was statistically significant as compared to the group $\mathrm{C}$.

- When group $\mathrm{S}+\mathrm{D}+\mathrm{AG}$ is compared with group $\mathrm{S}$, increase in the retention of CAR was statistically significant as compared to the group $\mathrm{S}$.

- When group $\mathrm{S}+\mathrm{D}+\mathrm{AG}$ is compared with group $\mathrm{C}$, increase in the retention of CAR was statistically significant as compared to the group $\mathrm{C}$.

- When group $\mathrm{S}+\mathrm{D}$ is compared with group $\mathrm{S}+\mathrm{AG}$, difference in the retention of CAR was not statistically significant between the two groups.

- When group $\mathrm{S}+\mathrm{AG}$ is compared with group $\mathrm{S}+\mathrm{AG}+\mathrm{D}$, difference in the retention of CAR was statistically significant between the two groups.

- When group $\mathrm{S}+\mathrm{D}$ is compared with group $\mathrm{S}+\mathrm{AG}+\mathrm{D}$, difference in the retention of CAR was not statistically significant between the two groups.

- When group $\mathrm{S}+\mathrm{AG}$ and $\mathrm{S}+\mathrm{D}$ is compared with group $\mathrm{S}+\mathrm{AG}+\mathrm{D}$, difference in the retention of CAR was statistically significant between the two groups.

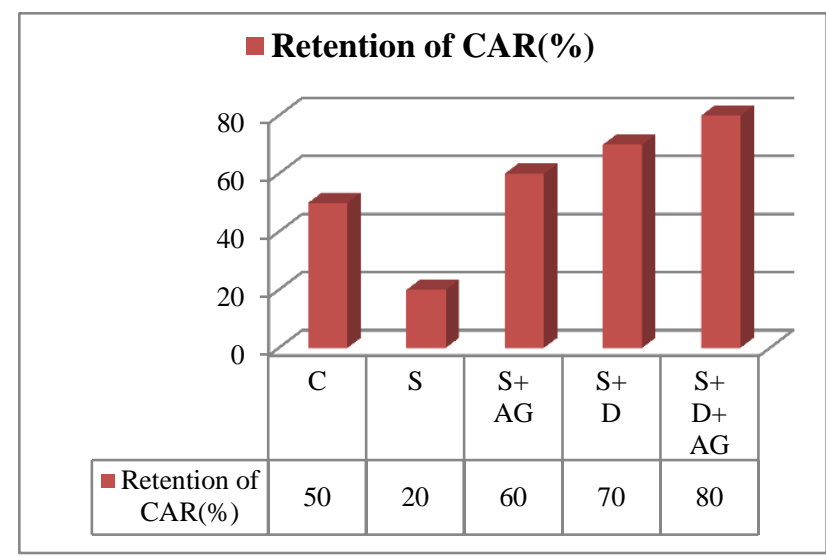

Figure 1: The comparison of all groups is shown in the graph. Distribution of groups is shown on the $\mathrm{X}$-axis and percentage of retention of CAR in each group is shown on the $\mathrm{Y}$-axis.

When the groups of study drugs i.e. $\mathrm{S}+\mathrm{AG}, \mathrm{S}+\mathrm{D}$, $\mathrm{S}+\mathrm{D}+\mathrm{AG}$, are compared with the scopolamine group (Group $S$ ) increase in the retention of CAR in groups $\mathrm{S}+$ AG, S+D, S+D+AG, is statistically significant.

When the combination group of $\mathrm{S}+\mathrm{D}+\mathrm{AG}$ is compared with the group $\mathrm{S}+\mathrm{AG}$ and $\mathrm{S}+\mathrm{D}$, there is statistically significant increase in the retention of CAR in the group $\mathrm{S}+\mathrm{D}+\mathrm{AG}$ when compared to the other two groups $(\mathrm{p}<0.05)$.

\section{DISCUSSION}

Dementia associated with probable $\mathrm{AD}$, is one of the most common types of dementia. Patients with AD often have cholinergic deficits in association with the disease. The cholinesterase inhibitors Donepezil hydrochloride, Galantamine hydrobromide and Rivastigmine tartrate are the current mainstays of symptomatic treatment for patients with $\mathrm{AD}$. In the clinical trials for all three agents, beneficial effects on standard measures of cognitive and global function have been observed in the patients with mild to moderate AD. Although none of the ChE inhibitors has been approved for treatment of patients in the advanced stages of $\mathrm{AD}$, all three agents have had beneficial cognitive effects among the patients with less severe forms of the disease.

Although there is no cure for the dementia of $\mathrm{AD}$ type, several available pharmacologic treatment modalities can reduce the symptoms of cognitive impairment, and slow the disease progression. Such treatments can increase the number of probable $\mathrm{AD}$ cases; classified as mild, potentially slowing or preventing the progression of the disease to moderate or severe. It is hoped that these modalities will allow for a shift away from the institutional care for these patients, and that their use may ease the public health burden of dementia of the $\mathrm{AD}$ type, as patients with mild $\mathrm{AD}$ exhibit less aberrant behaviour, and are allowed to function more independently than the patients with moderate or severe disease.

On the basis of evidence accumulated in the several $3-6$ month, randomized, double blind, placebo controlled trials, the American Academy of Neurology recommends the use of cholinesterase inhibitors as standard therapy for the patients with mild to moderate $\mathrm{AD} .{ }^{14}$

Working memory is a form of short-term memory with a limited capacity and an extremely rapid decay. Its impairment is more depictive of memory disorder in the Alzheimer's dementia. Although multiple neurotransmitters are involved in cognitive deficits, the most striking and consistent change is observed in the central cholinergic system. ${ }^{13}$

The present study was done to evaluate and to compare the role of American ginseng with Donepezil. We evaluated both the drugs individually and also studied the modulation of cognition by combining both drugs on the working memory in rats. We used the retention of CAR paradigm scopolamine induced disruption of memory in the rats.

This study was designed to compare the effects of American ginseng and Donepezil on the working memory in rats using scopolamine induced memory disruption model. Both the drugs were well tolerated, and there were no deaths during the study. The results showed that both American ginseng and Donepezil are equally 
effective in preventing the memory loss, and there is additional benefit by combining the two drugs as shown in the model.

Both these drugs have completely different profile regarding the mechanism of action, so the combination may be useful if given for a longer period of time and this warrants further highly specified animal experiments, and also needs to be evaluated in humans.

\section{CONCLUSION}

From the present study it can be concluded that:

- American ginseng and Donepezil are effective in the retention of working memory in rats.

- American ginseng is as effective as Donepezil in the retention of working memory in rats.

- The combination of American ginseng and Donepezil can have additive effect in the retention of working memory in rats.

Thus, results from this study provide a good platform for the benefit of combining American ginseng with Donepezil, but further studies with large sample size are required.

Funding: No funding sources Conflict of interest: None declared

Ethical approval: The study was approved by the Institutional Ethics Committee

\section{REFERENCES}

1. Parle M, Singh N, Vasudevan M. Regular rehearsal helps in consolidation of long term memory. J Sports Sci Med. 2006 Mar;5(1):80-8.

2. Masterman DL. Role of cholinesterase inhibitors in managing behavioural problems in Alzheimer's disease. Prim Care Companion J Clin Psychiatry. 2004;6(3):126-31.

3. Miranda MI, Ferreira G, Ramírez- LL, BermúdezRF. Role of cholinergic system on the construction of memories: taste memory encoding. Neurobiol Learn Mem. 2003 Nov;80(3):211-22.

4. Raded K, Gille G, Liu L, Rousch W. Use of Ginseng in Medicine with Emphasis on Neurodegenerative Disorders, J Pharmacol Sci. 2006;100:175-86.

5. Scholey A, Ossoukhova A, Owen L, Ibarra A, Pipinges A, He K et al. Effects of American ginseng (Panax quinquefolius) on neurocognitive function: An acute, randomized, double blind, placebo controlled, crossover study. Psychopharmacology. 2010;212:345-56.

6. Shah J, Goyal R. Comparative clinical evaluation of herbal formulation with multivitamin formulation for learning and memory enhancement. Asian J Pharm Clin Res. 2010 Jan-Mar;3(1):69-75.

7. Blokland A. Scopolamine induced deficits in cognitive performance: a review of animal studies. Scopolamine Rev. 2005 Jan;1:1-76.

8. Naveen K, Kohli K. Effect of metoclopramide on scopolamine induced working memory impairment in rats. Indian J Pharmacol. 2003 Mar-Apr;35 (2):104-8.

9. Castellano C, Cabib S, Puglisi-Allegra S. Psychopharmacology of memory modulation: evidence for multiple interactions among neurotransmitters and hormones. Behav Brain Res. 1996 May;77(1-2):1-21.

10. Khanzode SD, Belorkar NR, Gosavi DD. Effect of pretreatment of nimodipine and nitrendipine on some dopaminergically mediated behaviour in rats. Indian $\mathbf{J}$ Pharmacol. 2000 May-Jun;32(3):235-8.

11. Furey ML, PietriniP, Haxby JV, Drevets WC. Selective effects of cholinergic modulation on stask performance during selective attention. Neuropsychopharmacology. 2008 Mar;33(4):913-23.

12. Ghosh MN. Toxicity Studies. Fundamentals of experimental pharmacology. Kolkata, Ghosh SK and others; 2005:192.

13. Perry E, McKeith I, Ballard C. Butyryl cholinesterase and progression of cognitive deficits in dementia with Lewy bodies. Neurology. 2003 Jun;60(11):1852-3.

14. Baddeley AD. Working Memory. Science. 1992 Jan;255(5044):556-9.

Cite this article as: Shende SS, Shinde KM, Tilak AV, Rane BT, Raveendran S, Dabhade SA. A comparative study of American ginseng (spp. Panax quinquefolius) and Donepezil on working memory in rats using scopolamine induced disruption of memory. Int J Basic Clin Pharmacol 2017;6:547-51. 